\title{
Promotion and Rowmotion
}

\author{
Jessica Striker and Nathan Williams
}

School of Mathematics, University of Minnesota, 206 Church St. SE, Minneapolis, MN 55455

\begin{abstract}
We present an equivariant bijection between two actions-promotion and rowmotion—on order ideals in certain posets. This bijection simultaneously generalizes a result of R. Stanley concerning promotion on the linear extensions of two disjoint chains and certain cases of recent work of D. Armstrong, C. Stump, and H. Thomas on noncrossing and nonnesting partitions. We apply this bijection to several classes of posets, obtaining equivariant bijections to various known objects under rotation. We extend the same idea to give an equivariant bijection between alternating sign matrices under rowmotion and under B. Wieland's gyration. Lastly, we define two actions with related orders on alternating sign matrices and totally symmetric self-complementary plane partitions.

Résumé. Nous présentons une bijection équivariante entre deux actions—promotion et rowmotion—sur les idéaux d'ordre dans certaines posets. Cette bijection généralise simultanément un résultat de R. Stanley concernant la promotion sur les extensions linéaire de deux chaînes disjointes et certains cas des travaux récents de D. Armstrong, C. Stump, et H. Thomas sur les partitions noncroisées et nonemboîtées. Nous appliquons cette bijection à plusieurs classes de posets pour obtenir des bijections équivariantes a des différents objets connus sous la rotation. Nous généralisons la même idée pour donné une bijection équivariante entre les matrices à signes alternants sous rowmotion et sous la gyration de B. Wieland. Finalement, nous définissons deux actions avec des ordres similaires sur les matrices à signes alternants et les partitions plane totalement symétriques et autocomplémentaires.
\end{abstract}

Keywords: poset, order ideal, noncrossing, promotion, equivariant, alternating sign matrices

\section{Introduction}

In his 2009 survey paper on promotion and evacuation [Sta09], R. Stanley gave an equivariant bijection between linear extensions of two disjoint chains $[n] \oplus[k]$ under promotion (Pro) and order ideals of the product of two chains $[n] \times[k]$ under an operation that we call rowmotion (Row). In 2011, D. Armstrong, C. Stump, and H. Thomas gave a uniformly-stated equivariant bijection between noncrossing partitions under Kreweras complementation and nonnesting partitions under Row [AST11]. In particular, the type $A$ part of their theorem can be interpreted as an equivariant bijection between linear extensions of $[2] \times[n]$ under Pro and order ideals of the type $A$ positive root poset $\Phi^{+}\left(A_{n}\right)$ under Row.

We present a new proof of these two equivariant bijections between linear extensions and order ideals by simultaneously generalizing them as a single statement about rc-posets-certain posets whose elements and covering relations fit into rows and columns.

This theorem gives an equivariant bijection between the order ideals of an rc-poset $\mathcal{R}$ under Pro and Row by interpreting promotion as an action on the columns of order ideals of $\mathcal{R}$ and rowmotion as an action on the rows. Armed with promotion, we obtain simple equivariant bijections from the order ideals 
of $[n] \times[k], J([2] \times[n])$, positive root posets of types $A$ and $B$, and $[2] \times[m] \times[n]$ under rowmotion to various known objects under rotation. We also apply this theory to alternating sign matrices and totally symmetric self-complementary plane partitions.

Note that this paper is an extended abstract. The full version has been accepted for publication in the European Journal of Combinatorics and is available on the arXiv [SW11].

\section{Background}

In 1972, M.-P. Schützenberger defined promotion as an action on linear extensions [Sch72]. We will denote promotion by Pro.

Definition 2.1 Let $\mathcal{L}$ be a linear extension of a poset $\mathcal{P}$ and let $\rho_{i}$ act on $\mathcal{L}$ by switching $i$ and $i+1$ if they are not the labels of two elements with a covering relation. We define the promotion of $\mathcal{L}$ to be $\operatorname{Pro}(\mathcal{L})=\rho_{n-1} \rho_{n-2} \cdots \rho_{1}(\mathcal{L})$.

In 1973, P. Duchet defined an action on hypergraphs [Duc74]. This action was generalized by A. Brouwer and A. Schrijver to an arbitrary poset in [BS74]. Because we will interpret the action as acting on rows, we will call it rowmotion. We will denote rowmotion by Row.

Definition 2.2 Let $\mathcal{P}$ be a poset, and let $I \in J(\mathcal{P})$. Then $\operatorname{Row}(I)$ is the order ideal generated by the minimal elements of $\mathcal{P}$ not in $I$.

The Cyclic Sieving Phenomenon was introduced by V. Reiner, D. Stanton, and D. White as a generalization of J. Stembridge's $q=-1$ phenomenon [RSW04].

Definition 2.3 (V. Reiner, D. Stanton, D. White) Let $X$ be a finite set, $X(q)$ a generating function for $X$, and $C_{n}$ the cyclic group of order $n$ acting on $X$. Then the triple $\left(X, X(q), C_{n}\right)$ exhibits the Cyclic Sieving Phenomenon (CSP) if for $c \in C_{n}, X(\omega(c))=|\{x \in X: c(x)=x\}|$, where $\omega: C_{n} \rightarrow \mathbb{C}$ is an isomorphism of $C_{n}$ with the $n$th roots of unity.

In his 2009 survey paper [Sta09], R. Stanley noted that there was an equivariant bijection between promotion and rowmotion on the product of two chains.

Theorem 2.4 (R. Stanley) There is an equivariant bijection between $\mathcal{L}([n] \oplus[k])$ under Pro and $J([n] \times$ $[k])$ under Row.

Figure 1 illustrates this theorem for the case $n=k=2$.

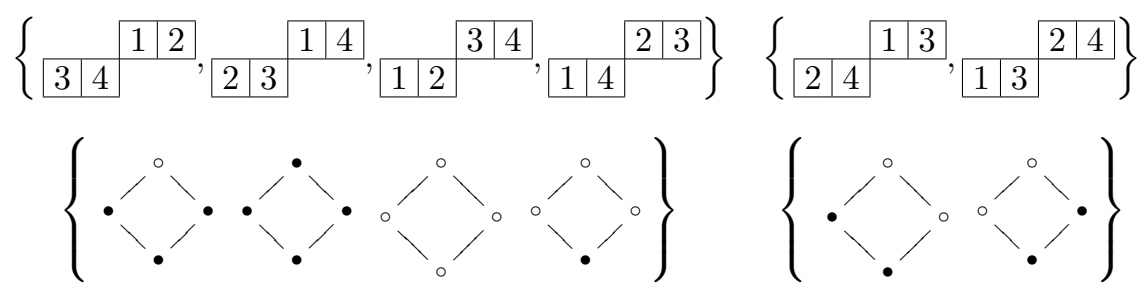

Fig. 1: The two orbits of $\mathcal{L}([2] \oplus[2])$ under Pro and of $J([2] \times[2])$ under Row. 
In 2007, D. Panyushev conjectured the order of rowmotion on order ideals of positive root posets $\Phi^{+}(W)$ [Pan09], which are well-known to be in bijection with nonnesting partitions of type $W$. D. Armstrong, C. Stump, and H. Thomas recently proved this conjecture in [AST11 by constructing an equivariant bijection from rowmotion on order ideals of a positive root poset to rotation of noncrossing matchings of the corresponding type [AST11]. The noncrossing matchings of type $W$ under rotation are known to have the order conjectured by D. Panyushev, are in equivariant bijection with Kreweras complementation on noncrossing partitions, and exhibit the CSP with a suitable $q$-analogue of the $W$ Catalan number, from which the results follow.

Theorem 2.5 (D. Armstrong, C. Stump, and H. Thomas) There is a uniformly-stated equivariant bijection between nonnesting partitions under rowmotion and noncrossing partitions under Kreweras complementation.

It is the noncrossing matchings that we can associate with linear extensions, using an unpublished result of D. White [Rho10, PPR09].

Theorem 2.6 (D. White) An equivariant bijection between type $A_{n}$ noncrossing matchings under rotation and SYT of shape $(n+1, n+1)$ under promotion is given by placing in the first row when it is the smaller of the two numbers in its matching.

In analogy with Theorem 2.4, we can restate the type $A_{n}$ result of Theorem 2.6 in the language of promotion.

Theorem 2.7 There is an equivariant bijection from $\mathcal{L}([2] \times[n+1])$ under Pro to $J\left(\Phi^{+}\left(A_{n}\right)\right)$ under Row.

Note that $\mathcal{L}([2] \times[n+1])$ are SYT of shape $(n+1, n+1)$. Figure 2 illustrates this theorem for $n=2$.

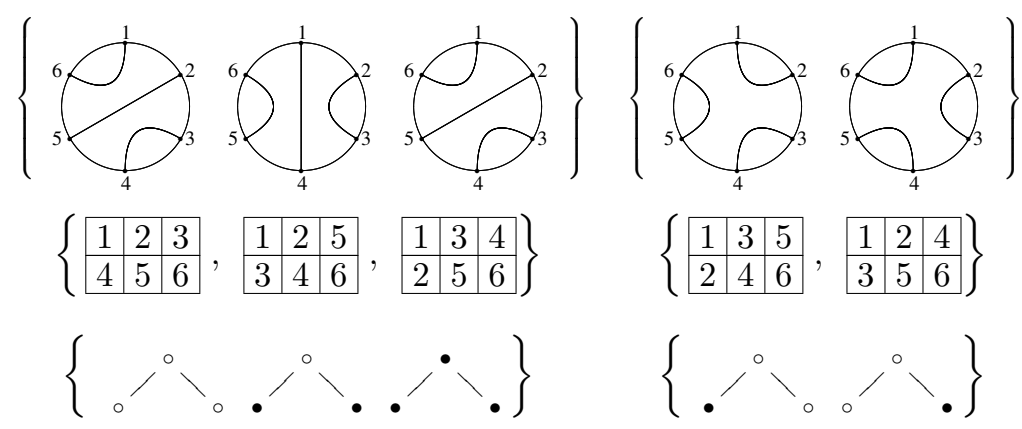

Fig. 2: The two orbits of noncrossing matchings on six points under rotation, the two orbits of SYT of shape $(3,3)$ under Pro and the two orbits of $J\left(\Phi^{+}\left(A_{2}\right)\right)$ under Row.

\section{Machinery}

In this section, we develop the machinery of the toggle group and rc-posets which we will need to prove our main theorem. We begin with the following lemma. 
Lemma 3.1 Let $G$ be a group acting on a set $X$, and let $g_{1}$ and $g_{2}=g g_{1} g^{-1}$ be conjugate elements in $G$. Then $x \rightarrow g x$ gives an equivariant bijection between $X$ under $\left\langle g_{1}\right\rangle$ and $X$ under $\left\langle g_{2}\right\rangle$.

\subsection{The Toggle Group}

Let $\mathcal{P}$ be a poset and let $J(\mathcal{P})$ be its set of order ideals. In [CFDF95], P. Cameron and D. Fon-der-Flaass defined a group acting on $J(\mathcal{P})$.

Definition 3.2 (P. Cameron and D. Fon-der-Flaass) For each $p \in \mathcal{P}$, define $t_{p}: J(\mathcal{P}) \rightarrow J(\mathcal{P})$ to act by toggling $p$ if possible. That is, if $I \in J(\mathcal{P})$,

$$
t_{p}(I)= \begin{cases}I \cup\{p\} & \text { if } p \notin I \text { and if } p^{\prime}<p \text { then } p^{\prime} \in I, \\ I-p & \text { if } p \in I \text { and if } p^{\prime}>p \text { then } p^{\prime} \notin I, \\ I & \text { otherwise. }\end{cases}
$$

The toggle group $T(\mathcal{P})$ is the subgroup of the permutation group $\mathfrak{S}_{J(\mathcal{P})}$ generated by $\left\{t_{p}\right\}_{p \in \mathcal{P}}$.

Note that $T(\mathcal{P})$ has the following obvious relations (which do not constitute a full presentation): $t_{p}^{2}=1$ and $\left(t_{p} t_{p^{\prime}}\right)^{2}=1$ if $p$ and $p^{\prime}$ do not have a covering relation.

P. Cameron and D. Fon-der-Flaass characterized rowmotion as an element of $T(\mathcal{P})$.

Theorem 3.3 (P. Cameron and D. Fon-der-Flaass) Fix a linear extension $\mathcal{L}$ of $\mathcal{P}$. Then $t_{\mathcal{L}^{-1}(1)} t_{\mathcal{L}^{-1}(2)} \cdots t_{\mathcal{L}^{-1}(n)}$ acts as Row.

\subsection{Rowed-and-Columned Posets}

We now define rc-posets-certain posets with elements that neatly fit into rows and columns and with covering relations allowed only between diagonally adjacent elements. We interpret promotion as an action that toggles the columns of order ideals of rc-posets and rowmotion as an action that toggles the rows.

Definition 3.4 Let $\Pi \subset \mathbb{R}^{2}$ be the set of points in the integer span of $(2,0)$ and $(1,1)$. A rowed-andcolumned ( $r c$ ) poset $\mathcal{R}$ is a finite poset together with a map $\pi: \mathcal{R} \rightarrow \Pi$, where if $p_{1}, p_{2} \in \mathcal{R}, p_{1}$ covers $p_{2}$, and $\pi\left(p_{1}\right)=(i, j)$, then $\pi\left(p_{2}\right)=(i+1, j-1)$ or $\pi\left(p_{2}\right)=(i-1, j-1)$. For $p \in \mathcal{R}$, we call $\pi(p) \in \Pi$ the position of $p$.

Let the height $h$ of an rc-poset be the maximum number of elements in a single position $(i, j)$. The $j$ th row of an rc-poset $\mathcal{R}$ is the set of elements of $\mathcal{R}$ in positions $\{(i, j)\}_{i}$. The $i$ th column of an rc-poset is the set of elements of $\mathcal{R}$ in positions $\{(i, j)\}_{j}$. Let $n$ denote the maximal non-empty row and $k$ the maximal non-empty column. For an example, see Figure 3 .

Definition 3.5 If $\mathcal{R}$ is an rc-poset, let $r_{i}=\prod t_{q}$, where the product is over all elements in row $i$ and let $c_{i}=\prod t_{q}$, where the product is over all elements in column $i$.

Then, since no elements within a row or column of an rc-poset share a covering relation, the following relations hold: $r_{i}^{2}=c_{i}^{2}=1$ and if $|i-j|>1,\left(r_{i} r_{j}\right)^{2}=\left(c_{i} c_{j}\right)^{2}=1$. 


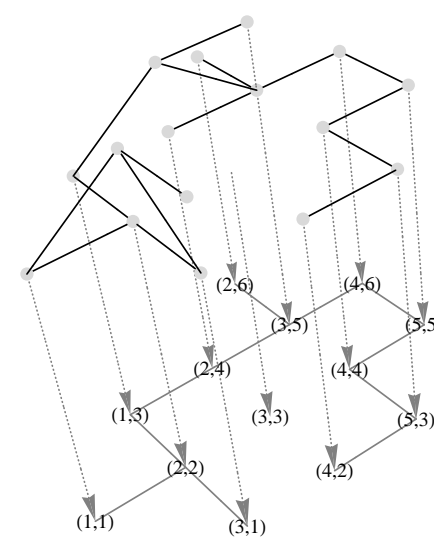

Fig. 3: This picture represents an rc-poset with height $h=2, k=5$ columns, and $n=6$ rows. When there are two elements with the same position, the second element is raised; the position is indicated by a dotted arrow down. Covering relations are drawn with solid black lines and are projected down as solid gray lines.

\subsection{Promotion and Rowmotion in the Toggle Group}

We interpret promotion and rowmotion as elements of the toggle group of an rc-poset with $n$ rows and $k$ columns. For ease of notation, we assume that the rc-poset is translated into the first quadrant so that the rows are labeled from 1 to $n$ and the columns from 1 to $k$.

Definition 3.6 Given $\nu \in \mathfrak{S}_{k}$ let $\operatorname{Pro}_{\nu}=\prod_{i=1}^{k} c_{\nu(i)}=c_{\nu(1)} \cdot c_{\nu(2)} \cdots c_{\nu(k)}$. Likewise, given $\omega \in \mathfrak{S}_{n}$ let $\operatorname{Row}_{\omega}=\prod_{i=1}^{n} r_{\omega(i)}$.

We now specify the element of the toggle group that acts as rowmotion, as a corollary of Theorem 3.3

Corollary 3.7 On rc-posets, $P_{12 \ldots n}$ acts as Row.

Interpreting promotion as an element of the toggle group takes slightly more work. Let $\mathcal{P}$ be a Ferrers diagram. Following R. Stanley in [Sta09], we define promotion using the order ideals $J(\mathcal{P})$. Linear extensions $\mathcal{L}$ can be interpreted as maximal chains $\emptyset=I_{0} \subset I_{1} \cdots \subset I_{n}=\mathcal{P}$ in $J(\mathcal{P})$ by taking $\mathcal{L}(p)=i$ if $p$ is the element in the singleton set $I_{i+1}-I_{i}$. The promotion of $\lambda=\left(\emptyset=I_{0} \subset I_{1} \cdots \subset I_{n}=\mathcal{P}\right)$ is $\tau_{n-1} \cdots \tau_{1} \lambda$, where $\tau_{i}$ acts on a chain by switching $I_{i}$ to the other order ideal in the interval $\left[I_{i-1}, I_{i+1}\right]$, if one exists. Figure 4 illustrates promotion on the maximal chains.

When $\lambda / \mu=(n+k, m) /(k)$ is a skew Ferrers diagram with at most two rows, we can draw the Hasse diagram of $J(\lambda / \mu)$ as an rc-poset. The $i$ th step of a maximal chain in $J(\lambda / \mu)$ is taken to be northwest if the corresponding linear extension of $\lambda / \mu$ associates $i$ to an element in the first row, and northeast otherwise. We take advantage of this planarity with the following definition.

Definition 3.8 If $\lambda / \mu=(n+k, m) /(k)$ is a skew Ferrers diagram with at most two rows, define the interior $\operatorname{Int}(J(\lambda / \mu))$ to be the rc-poset with elements the boxes of $J(\lambda / \mu)$ and covering relations between two elements when their corresponding boxes are adjacent. 


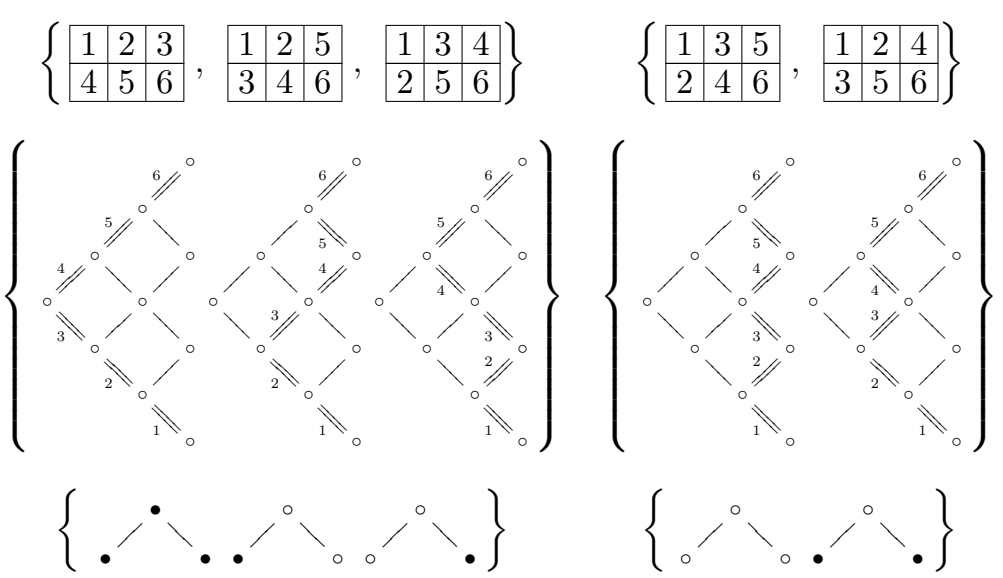

Fig. 4: The two orbits of SYT of shape $(3,3)$ under promotion, the same two orbits as maximal chains, and the same two orbits as order ideals under Pro.

When $\lambda / \mu$ has at most two rows, a maximal chain in $J(\lambda / \mu)$ traces out an order ideal—defined by the boxes to the right of the maximal chain-in $\operatorname{Int}(J(\lambda / \mu))$. Figure 4 illustrates this bijection.

Theorem 3.9 Let $\mathcal{P}=(n+k, m) /(k)$ be a skew Ferrers diagram with at most two rows. Then there is an equivariant bijection from $\operatorname{Int}(J(\mathcal{P}))$ under $\operatorname{Pro}_{k \ldots 21}$ to SYT of shape $\mathcal{P}$ under ordinary promotion.

We now extend the definition of promotion from order ideals of rc-posets that correspond to skew SYT to order ideals of any rc-posets. We also generalize the maximal chains by defining boundary paths.

Definition 3.10 Given an rc-poset $\mathcal{R}$ and an order ideal $I \in J(R)$, define the promotion of $I$ to be $\operatorname{Pro}(I)=\operatorname{Pro}_{k \ldots 21}(I)$.

Definition 3.11 We define the boundary path of an order ideal of a connected rc-poset of height one to be the path that separates the order ideal from the rest of the poset. We encode boundary paths as binary words by writing a 1 for a northeast step and a 0 for a southeast step.

When we start with the poset $\Phi^{+}\left(A_{n}\right)$ under Pro and map to SYT as above, we can apply Theorem 2.6 to obtain noncrossing matchings under rotation. In this language, $i$ is the smaller number in its partition if the $i$ th step of the boundary path is northeast. We apply this idea of boundary paths under Pro to noncrossing objects under rotation in Section 5 , and generalize it in Section 6.

\section{The Conjugacy of Promotion and Rowmotion}

We now prove that promotion and rowmotion are conjugate elements in the toggle group of an rc-poset and then spend the rest of the paper applying this theorem to specific rc-posets.

Lemma 4.1 ([HH92]) Let $G$ be a group whose generators $g_{1}, \ldots, g_{n}$ satisfy $g_{i}^{2}=1$ and $\left(g_{i} g_{j}\right)^{2}=1$ if $|i-j|>1$. Then for any $\omega, \nu \in \mathfrak{S}_{n}, \prod_{i} g_{\omega(i)}$ and $\prod_{i} g_{\nu(i)}$ are conjugate.

Theorem 4.2 For any rc-poset $\mathcal{R}$ and any $\omega \in \mathfrak{S}_{n}$ and $\nu \in \mathfrak{S}_{k}$, there is an equivariant bijection between $J(\mathcal{R})$ under $\operatorname{Pro}_{\nu}$ and $J(\mathcal{R})$ under $\operatorname{Row}_{\omega}$. 
Proof: Since the row (resp. column) toggles $r_{i}$ (resp. $c_{i}$ ) satisfy the conditions of Lemmas 4.1 and 3.1. for any rc-poset $\mathcal{R}$ and any $\omega, \nu \in \mathfrak{S}_{n}$ (resp. $\mathfrak{S}_{k}$ ), there is an equivariant bijection between $J(\mathcal{R})$ under Row $_{\omega}$ (resp. $\operatorname{Pro}_{\omega}$ ) and $J(\mathcal{R})$ under $\operatorname{Row}_{\nu}\left(\right.$ resp. $\operatorname{Pro}_{\nu}$ ). Therefore, we may restrict to considering only Row $135 \ldots 246 \ldots$ and Pro $_{135} \ldots 246 \ldots$. But since all $t_{p}$ with Row in an odd (resp. even) column or row commute with one another, and since elements in an odd (resp. even) row are also necessarily in an odd (resp. even) column, we conclude that Row $135 \ldots 246 \ldots$ is equal to Pro $_{135 \ldots 246 \ldots}$.

\section{RC-Posets of Height One}

We apply Theorem 4.2 to the following rc-posets: $[n] \times[k], J([2] \times[n-1]), \Phi^{+}\left(A_{n}\right)$, and $\Phi^{+}\left(B_{n}\right)$.

\section{$5.1[n] \times[k]$}

As a corollary of Theorem 4.2 we obtain a new proof of Theorem 2.4 Since $\mathcal{L}([n] \oplus[k])$ under Pro is in bijection with $\left(\begin{array}{c}{[n+k]} \\ k\end{array}\right)$ under the cycle $(1,2, \ldots, n+k)$, we can restate the theorem using the map from Theorem 3.9

Theorem 5.1 There is an equivariant bijection between $I \in J([n] \times[k])$ under Row and binary words $w=w_{1} w_{2} \ldots w_{n+k}$ of length $n+k$ with $n$ l's under rotation.

The bijection is given by using our bijection from $J([n] \times[k])$ under Row to $J([n] \times[k])$ under Pro, and then setting the $w_{i}$ to 1 if the $i$ th step of the boundary path is northeast, and to 0 otherwise. The set of binary words of length $n+k$ with $n$ 1's exhibits the CSP under rotation with the polynomial $\left(\begin{array}{c}n+k \\ n\end{array}\right)_{q}$.

$5.2 J([2] \times[n-1])$

Observe that $J([2] \times[n-1])$ can be embedded as the left half of $[n] \times[n]$. It is not hard to see that the map from Theorem 3.9 can be adapted to these boundary paths.

Theorem 5.2 There is an equivariant bijection between $I \in J(J([2] \times[n-1]))$ under Row and binary words of the form $w(1-w)$ under rotation, where $w=w_{1} w_{2} \ldots w_{n}$ is a binary word of length $n$ and $1-w$ is the word of length $n$ whose ith letter equals $1-w_{i}$.

Again, we first use our bijection from $J(J([2] \times[n-1]))$ under Row to $J(J([2] \times[n-1]))$ under Pro, and then set $w_{i}$ equal to 1 if the $i$ th step of the boundary path is northeast, and 0 otherwise. This theorem is illustrated for the case $n=3$ in Figure 5 . The set of binary words of the form $w(1-w)$, where $w$ is a binary word of length $n$, exhibits the CSP under rotation with the polynomial $\prod_{i=1}^{n}[2]_{q^{i}}$.

\section{$5.3 \Phi^{+}\left(A_{n}\right)$}

Using D. White's equivariant bijection between $\mathcal{L}([2] \times[n+1])$ and noncrossing matchings, we obtain the type $A_{n}$ case of Theorem 2.5

\section{$5.4 \Phi^{+}\left(B_{n}\right)$}

The type $B_{n}$ case of Theorem 2.5 also follows from a modification of the map in Theorem 3.9 since $B_{n}$ noncrossing matchings are just the half-turn symmetric $A_{2 n-1}$ matchings. See Figure 6

Corollary 5.3 There is an equivariant bijection between type $B_{n}$ noncrossing matchings under rotation and $J\left(\Phi^{+}\left(B_{n}\right)\right)$ under Row. 


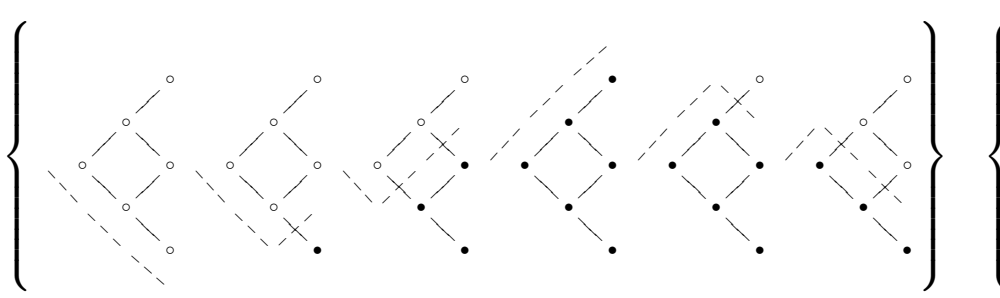

$\{000111,001110,011100,111000,110001,100011\}$

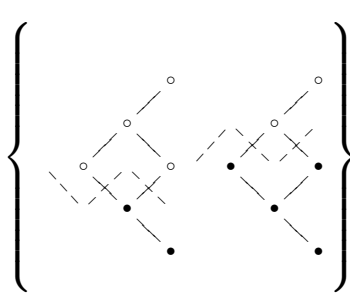

$\{010101,101010\}$

Fig. 5: There are two orbits of $J(J([2] \times[2]))$ under Pro (the dashed lines are the boundary paths corresponding to the order ideals) and two orbits of binary words of length 6 of the form $w(1-w)$ under rotation (obtained from the boundary paths).
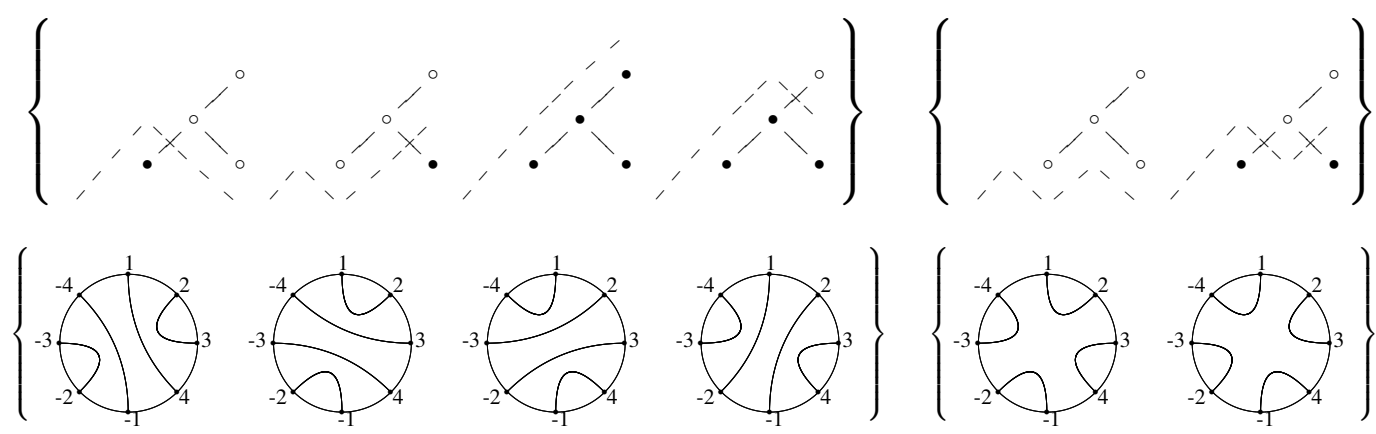

Fig. 6: The two orbits of $J\left(\Phi^{+}\left(B_{2}\right)\right)$ under Pro (the dashed lines are the boundary paths corresponding to the order ideals) and the two orbits of type $B_{2}$ noncrossing matchings under rotation (obtained from the boundary path by taking $i$ to be the smaller element of its block if the $i$ th step was northeast).

\section{Plane Partitions}

Consider the order ideals of the product of three chains - that is, plane partitions - under rowmotion. We draw $[\ell] \times[m] \times[n]$ as an rc-poset of height $\ell$ to generalize the approach in Theorem 3.9 , simplifying proofs due to P. Cameron and D. Fon-der-Flaass and D. B Rush and X. Shi.

V. Reiner originally conjectured that $\left(J([2] \times[m] \times[n]), M_{2, m, n}(q), C_{m+n+1}\right)$ exhibited the CSP. D. B Rush and X. Shi recently proved this using P. Cameron and D. Fon-der-Flaass's equivariant bijection to parenthesized words [RS11]. Their theorem, which we obtain as a corollary, was the inspiration for our bijection to noncrossing partitions in Theorem 6.2

As usual, we immediately obtain the following corollary of Theorem 4.2 .

Corollary 6.1 There is an equivariant bijection between $J([\ell] \times[m] \times[n])$ under Row and $J([\ell] \times[m] \times$ $[n])$ under Pro.

Theorem 6.2 There is an equivariant bijection between $J([2] \times[m] \times[n])$ under Row and noncrossing partitions of $[n+m+1]$ into $m+1$ blocks under rotation. 


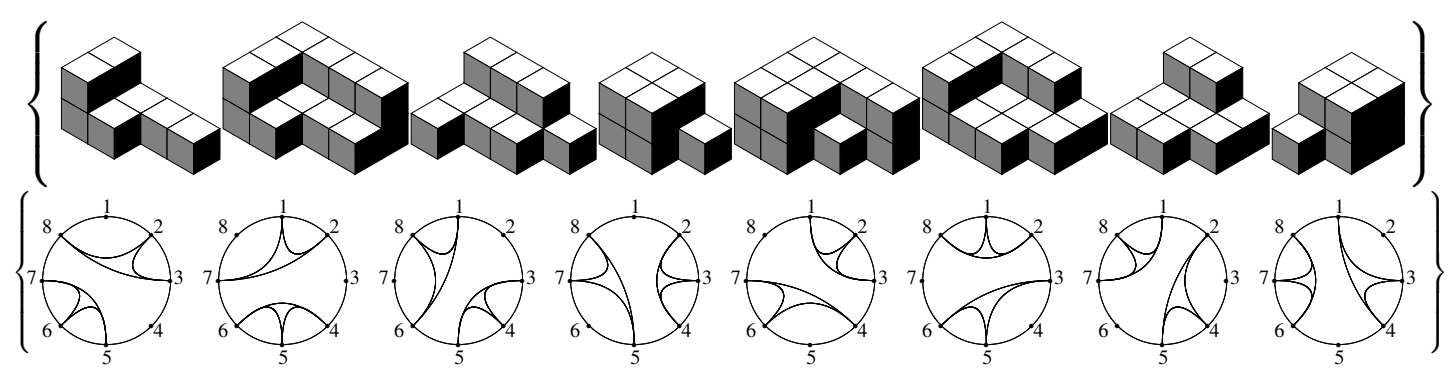

Fig. 7: An orbit of $J([2] \times[3] \times[4])$ under promotion (drawn using code written by J. S. Kim for TikZ) and the corresponding noncrossing partitions.

Corollary 6.3 (D. Rush, X. Shi) Let $C_{m+n+1}$ act on $J([2] \times[m] \times[n])$ by Row. Then $(J([2] \times[m] \times$ $\left.[n]), M_{2, n, m}(q), C_{m+n+1}\right)$ exhibits the CSP.

\section{ASMs and TSCCPPs}

We apply our methods to the alternating sign matrix and totally symmetric self-complementary plane partition posets. In particular, we define two actions with related orders on ASMs and TSSCPPs, with the open problem of finding an explicit bijection between these two sets of objects as our motivation. Additionally, we give an equivariant bijection between ASMs under rowmotion under B. Wieland's gyration,

An alternating sign matrix (ASM) of order $n$ is an $n \times n$ matrix with entries 0,1 , or -1 whose rows and columns sum to 1 and whose nonzero entries in each row and column alternate in sign. We begin by recalling the poset interpretation of ASMs, first introduced in [LS96] by A. Lascoux and M.P. Schützenberger. This poset is usually defined using monotone triangles, but we choose to define it equivalently using height functions because of the connection with gyration later in this section. Height functions of order $n$ are in bijection with $n \times n$ ASMs.

Definition 7.1 $A$ height function of order $n$ is an $(n+1) \times(n+1)$ matrix $\left(h_{i, j}\right)_{0 \leq i, j \leq n}$ with $h_{0, k}=$ $h_{k, 0}=k$ and $h_{n, k}=h_{k, n}=n-k$ for $0 \leq k \leq n$, and such that adjacent entries in any row or column differ by 1.

Height functions of order $n$ have a partial ordering given by componentwise comparison of entries. This poset is a distributive lattice which is the MacNeille completion of the Bruhat order on the symmetric group [LS96]. We denote the poset of join irreducibles as $\mathbf{A}_{n}$, so that $J\left(\mathbf{A}_{n}\right)$ is in bijection with the set of $n \times n$ ASMs. See [Str11] for further discussion.

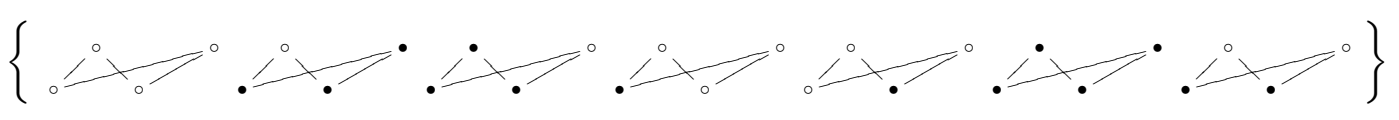

Fig. 8: The seven order ideals in $J\left(\mathbf{A}_{3}\right)$. They form a single orbit under superpromotion.

Figure 8 gives the order ideals of $\mathbf{A}_{3}$. We can draw $\mathbf{A}_{n}$ as an rc-poset of height $h=n-1$ (see Figure 9 for an example and [SW11] for a detailed explanation), which gives the following corollary of Theorem 4.2 
Corollary 7.2 There is an equivariant bijection between $J\left(\mathbf{A}_{n}\right)$ under Row and under Pro.
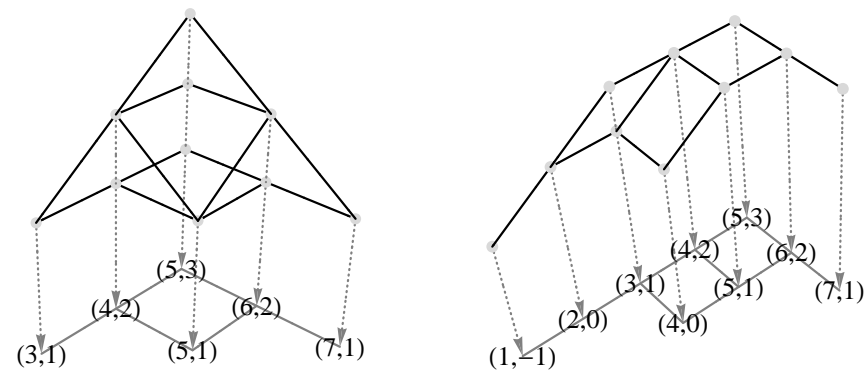

Fig. 9: Left: $\mathbf{A}_{4}$ drawn as an rc-poset of height 3. Right: $\mathbf{T}_{4}$ drawn as an rc-poset of height 2. When there are multiple elements with the same position, subsequent elements are raised; the position is indicated by a dotted arrow down. Covering relations are drawn with solid black lines, and are projected down as solid gray lines.

Similarly, we denote the poset whose order ideals are in bijection with TSSCPPs as $\mathbf{T}_{n}$. We can draw $\mathbf{T}_{n}$ as an rc-poset of height $\left\lfloor\frac{n}{2}\right\rfloor$. See Figure 9 for an example and [SW11] for the details. Note that this partial order on TSSCPPs is the same as the partial order on the magog triangles of [Zei96]. Figure 10 gives the order ideals of $\mathbf{T}_{3}$.

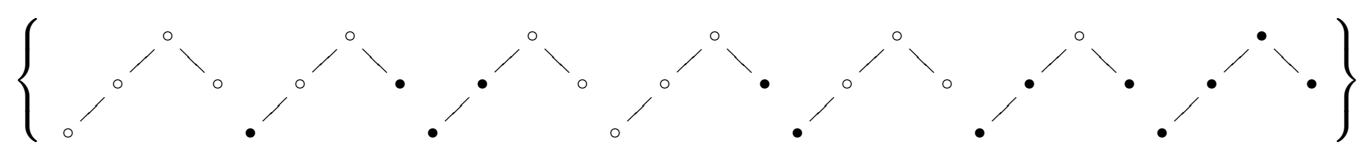

Fig. 10: There are seven order ideals in $J\left(\mathbf{T}_{3}\right)$. They form a single orbit under rowmotion.

Appealing once again to Theorem 4.2, we obtain the conjugacy of Row and Pro.

Corollary 7.3 There is an equivariant bijection between $J\left(\mathbf{T}_{n}\right)$ under Row and under Pro.

Promotion acts on the empty order ideal with order $3 n-2$ (see [SW11]), so we have the following theorem.

Theorem 7.4 $J\left(\mathbf{A}_{n}\right)$ under Row has order divisible by $3 n-2$.

In [SW11], we define a related action on the ASM poset called superpromotion-denoted SPro. Superpromotion acts by performing promotion successively in each layer of $\mathbf{A}_{n}$; we show that its order is also a multiple of $3 n-2$. Since the order of Pro on $J\left(\mathbf{T}_{n}\right)$ and of SPro on $J\left(\mathbf{A}_{n}\right)$ are related, one could hope to define a bijection from ASMs to TSSCPPs using a noncrossing combinatorial object with $3 n-2$ external vertices, such that these actions translate to rotation of those vertices.

Interestingly, a conjugate to Pro and Row in the toggle group of $\mathbf{A}_{n}$ has already been studied.

Definition 7.5 Consider the grid $[n] \times[n]$. A fully-packed loop configuration (FPL) of order $n$ is a set of paths that begin and end only at every second outward-pointing edge, such that each of the $n^{2}$ vertices within the grid lie on exactly one path. 
Figure 11 gives the FPLs of order 3. FPLs of order $n$ are in bijection with $n \times n$ ASMs.

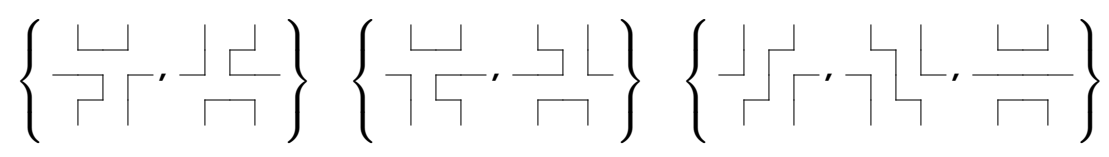

Fig. 11: The seven FPLs of order 3. They break into three orbits under gyration.

Definition 7.6 Pairing up the boundary edges of each path reduces the FPL to a noncrossing matching on $2 n$ vertices. This matching is called the link pattern of the FPL.

In 2000, B. Wieland defined an action called gyration on FPLs, which he proved rotated the corresponding link pattern [Wie00]. In 2010, L. Cantini and A. Sportiello generalized gyration in their proof of the Razumov-Stroganov conjecture that the number of FPLs with a given link pattern appear as the groundstate components of the $O(1)$ loop model of statistical physics [CS10].

Definition 7.7 Given an FPL, its gyration is computed by first visiting all squares with lower left-hand corner $(i, j)$ for which $i+j$ is even, and then all squares for which $i+j$ is odd, swapping the edges around a square if the edges are parallel and otherwise leaving them fixed.

Proposition 7.8 Gyration acts on height functions $\left(h_{i, j}\right)_{0 \leq i, j \leq n}$ by visiting all entries $h_{i, j}$, first those for which $i+j$ is even, and then those for which $i+j$ is odd, changing $h_{i, j}$ to its other possible value if each adjacent entry is equal and otherwise leaving it fixed.

We may now interpret gyration directly in terms of the toggle group of the poset $\mathbf{A}_{n}$.

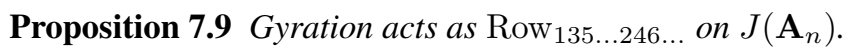

Therefore, by Lemma 4.1, we conclude that rowmotion and gyration are conjugate elements.

Theorem 7.10 There is an equivariant bijection between $J\left(\mathbf{A}_{n}\right)$ under rowmotion and under gyration.

\section{Acknowledgments}

The authors thank Vic Reiner, Dennis Stanton, and Dennis White for many helpful conversations and suggestions, and Peter Webb for his insights regarding toggle groups. They are particularly grateful to David B Rush and Xiaolin Shi for their work on plane partitions, to Vic Reiner for introducing them to rowmotion, and to the anonymous reviewers for their many helpful comments. 


\section{References}

[AST11] D. Armstrong, C. Stump, and H. Thomas. A uniform bijection between nonnesting and noncrossing partitions. Arxiv preprint arXiv:1101.1277v2[math.CO], January 2011.

[BS74] A. Brouwer and A. Schrijver. On the period of an operator, defined on antichains. Math Centrum report ZW, 24/74, 1974.

[CFDF95] P. Cameron and D. Fon-Der-Flaass. Orbits of antichains revisited. European J. Combin., 16(6):545-554, 1995.

[CS10] L. Cantini and A. Sportiello. Proof of the razumov-stroganov conjecture. Arxiv preprint arXiv:1003.3376v1 [math.CO], 2010.

[Duc74] P. Duchet. Sur les hypergraphes invariants. Discrete Math., 8(3):269-280, 1974.

[HH92] P. Hoffman and J. Humphreys. Projective representations of the symmetric groups. Oxford Mathematical Monographs. The Clarendon Press Oxford University Press, New York, 1992. $Q$-functions and shifted tableaux, Oxford Science Publications.

[LS96] A. Lascoux and M. Schützenberger. Treillis et bases des groupes de Coxeter. Electron. J. Combin., 3(2), 1996.

[Pan09] D. Panyushev. On orbits of antichains of positive roots. European J. Combin., 30(2):586-594, 2009.

[PPR09] T. Petersen, P. Pylyavskyy, and B. Rhoades. Promotion and cyclic sieving via webs. J. Algebraic Combin., 30(1):19-41, 2009.

[Rho10] B. Rhoades. Cyclic sieving, promotion, and representation theory. J. Combin. Theory Ser. A, 117(1):38-76, 2010.

[RS11] D. B. Rush and X. Shi. On orbits of order ideals of minuscule posets. Arxiv preprint arXiv:1108.5245 [math.CO], 2011.

[RSW04] V. Reiner, D. Stanton, and D. White. The cyclic sieving phenomenon. J. Combin. Theory Ser. A, 108(1):17-50, 2004.

[Sch72] M.-P. Schützenberger. Promotion des morphismes d'ensembles ordonnés. Discrete Math., 2(1):73-94, 1972.

[Sta09] R. Stanley. Promotion and evacuation. Electron. J. Combin., 16(2):R9, 2009.

[Str11] J. Striker. A unifying poset perspective on alternating sign matrices, plane partitions, catalan objects, tournaments, and tableaux. Adv. Appl. Math., 46(1-4):583-609, 2011.

[SW11] J. Striker and N. Williams. Promotion and rowmotion. Arxiv preprint arXiv:1108.1172, 2011.

[Wie00] B. Wieland. A large dihedral symmetry of the set of alternating sign matrices. Electron. $J$. Combin., 7:Research Paper 37, 13 pp. (electronic), 2000. 
[Zei96] D. Zeilberger. Proof of the alternating sign matrix conjecture. Electron. J. Combin., 3(2):Research Paper 13, approx. 84 pp. (electronic), 1996. The Foata Festschrift. 